\title{
Neutron powder diffraction advances
}

With this fourth Issue of Powder Diffraction journal in 2017, it is exciting to publish the Technical Article "Calculation of Full-Pattern Neutron Time-of-Flight (TOF) Powder Diffraction Patterns" authored by Dr. John Faber. This paper is particularly relevant due to the advances in neutron sources, particularly spallation neutron sources. Advanced spallation neutron sources such as ISIS (England), J-PARC (Japan), and SNS (USA) and the under-construction ESS (Sweden) and CSNS (China) are or will be providing extended user access, even by mail in service. These new facilities complement the existing high-flux reactor-based scattering centers (many more than spallation facilities).

Neutron diffraction complements X-ray diffraction for the determination of the atomic and/or magnetic structure of a material or for the in situ studies of changes in structure or phases as a function of temperature, pressure, and/or environment. Neutrons have a better sensitivity to light atoms (low $Z$ elements), to isotopes of each element, as well as exhibiting low radiation damage and a larger penetration depth (several $\mathrm{cm}$ ). The good penetration depth enables studies of active components such as advanced batteries undergoing cyclic charging and discharging or the phase reactions while temperature or pressure is varying. Because of these advanced neutron sources and their associated user programs with easy and rapid access mechanisms, the use of neutron powder diffraction is expanding worldwide.

The Technical Article by Dr. John Faber describes another advance that will support users who are considering neutron powder diffraction. His paper shows that a user of the current version of PDF4+ $\left(\mathrm{ICDD}^{\circledR}\right.$, proprietor of this journal) has access to new capabilities via the embedded software to simulate neutron TOF patterns (those obtained at spallation sources). Pattern calculation capabilities for constant wavelength neutron sources were implemented a couple years ago. In addition, with the new TOF pattern calculating capabilities coupled with the nearly 300000 atomic structures in the PDF4+, one can now generate a Search/Match database for a particular neutron instrument so that if new phases arise during an in situ experiments or unexpected extra peaks are observed from a sample, a PDF4+ ${ }^{\mathrm{TM}}$ Search/Match can be employed to characterize the new phase or the unexpected peaks. Similarly, a quantitative phase analysis is also possible.

This paper also demonstrates that the simulation of the neutron background from a sample can be computed. This can be particularly valuable to scientists considering the use of neutrons in that background levels, which can be quite high if hydrogen is present, can be productively reduced by replacement with deuterium. Knowing the background to be expected can play a major role in estimating measurement time or even success of a proposed neutron diffraction experiment.

The journal Powder Diffraction looks forward to receiving an increasing number of papers based on the use of neutron powder diffraction methods for materials characterization, structure analysis, and in situ studies.

Camden Hubbard

Editor-in-Chief

Email: camden.hubbard@me.com 